\title{
intuitio
}

\section{RADICAL LIBERTAD HUMANA: CONDENA Y PRÁCTICA DE LA LIBERTAD EN LA LECTURA DE FREIRE A SARTRE}

\author{
RADICAL HUMAN FREEDOM: CONDEMNATION AND \\ PRACTICE OF FREEDOM IN A READING OF FREIRE TO SARTRE
}

Diego Alejandro Muñoz Gaviria'

\begin{abstract}
Resumen: La lectura de Paulo Freire a Jean Paul Sartre parte del reconocimiento del ser humano como un ser condenado a la libertad. A diferencia de otras especies, la humana sólo encuentra su realización en la libertad. Ser libre es asumir la responsabilidad ética y política de hacer algo de la propia vida, reconocerse como ser responsable que asume la posibilidad de su existencia como un compromiso con todo lo viviente. Para los dos autores, la libertad no podría ser una donación o regalo de otros, es la defensa y conquista de la dignidad humana en cuanto realización como especie. Las implicaciones de estas tesis filosóficas y políticas de Sartre en la pedagogía de Freire permiten comprender la educación como práctica de la libertad.
\end{abstract}

Palabras clave: Libertad. teoría crítica. Emancipación. Educación. Formación. radicalidad. Abstract: The reading of Paulo Freire by Jean Paul Sartre starts from the recognition of the human as a condemned to freedom. Unlike other species, only humans finds its fulfillment in freedom. To be free is to assume the ethical and political responsibility to do something of one's own life, to recognize oneself as a responsible that assumes the possibility of its existence as a commitment to all living things. For the two authors, freedom could not be a donation or gift from others, it is the defense

\footnotetext{
1 Miembro del Grupo SER y profesor de la facultad de Ciencias de la educación de la Universidad Católica de Oriente (UCO). ORCID: http://orcid.org/o000-0003-0480-9723. E-Mail: diegomudante@gmail.com
} 
and conquest of human dignity in terms of realization as a species. The implications of these philosophical and political theses of Sartre in Freire's pedagogy allow us to understand education as a practice of freedom.

Keywords: Freedom. critical theory. Emancipation. Education. Formation. radicality.

\section{A MODO DE INTRODUCCIÓN:}

En este texto se propone una lectura filosófica - política a la apropiación que el pedagogo brasileño Paulo Freire (1921 - 1997) hace de la obra del filosofo francés Jean Paul Sartre (1905 - 1980), con especial interés en la concepción de libertad sustentada por el brasileño en sus dos primeros capítulos del texto Pedagogía del Oprimido (1970)² donde retoma con suma precisión la obra del Francés: El Hombre y Las Cosas (1965). La tesis a sustentar es que en la idea de libertad de Freire, es decir, en la comprensión del ser humano como ser condenado a la libertad y por ello obligado a tener que decidirse, se encuentra el eco sartreano. Ese eco será enunciado en lo sucesivo como la radical libertad humana, una interpretación de la pregunta por el ser humano, su radicalidad ${ }^{3}$, en clave de libertad. La condición humana sólo tiene sentido Biológico, biográfico e histórico desde su condena a la libertad. Sin el presupuesto de la libertad no se podría comprender la humana condición (Estrada, 2018).

En las siguientes páginas se reconstruirán las principales implicaciones o ecos sartrianos en la concepción radical de libertad de Freire expuesta en sus primeros capítulos de la Pedagogía Del Oprimido, para ello se partirá de las apropiaciones de la Teoría Crítica en el pedagogo para luego exponer sus tesis en torno a la radical libertad.

\footnotetext{
2 La versión aquí consultada es la de 1983.

3 "Ser radical es coger el problema por la raíz. Pero la raíz para el hombre es el hombre mismo" (Marx, 2008, p.103)
} 


\section{APROPIACIONES DE LA TEORÍA CRÍTICA EN LA OBRA DE PAULO FREIRE}

En los estudios de apropiaciones, en donde se inscribe este trabajo, autores como Roger Chartier (2000) exponen la importancia de reconstruir las condiciones históricas tanto del contexto de producción de una obra como del contexto de apropiación. Si se hiciera caso en la totalidad de esta sugerencia metodológica se necesitarían más páginas de las aquí presentadas, sin embargo, y con el interés de contextualizar las obras, se puede decir a grande líneas que tanto el trabajo de Sartre como el de Freire se inscriben en la realidad histórica de la segunda mitad del siglo $\mathrm{XX}$, principalmente en las condiciones derivadas de la segunda guerra mundial, la guerra fría, los movimientos libertarios en el tercer mundo, los nuevos movimientos sociales y la expansión neoliberal del capitalismo. La inquietud por la libertad de Sartre y de Freire se inscriben en tanto condiciones de época, en la crítica e intento de transformación de unas realidades globales instrumentalizantes, apoyadas en la falsa idea de libertad defendida en clave neoliberal por la sociedad del mercado. Podría decirse que la lectura que Freire desarrolla a la obra de Sartre se orienta por el interés compartido de luchar contra el imperio único del capital y sus situaciones y prácticas de opresión, la mayoría de la veces, disfrazadas de "libertad". En efecto fenómenos que demuestran la insignificancia de la decisión como las opciones consumistas, la reducción caricaturesca de la democracia a la participación en la industria cultural o la confusa realización del ser humano en tanto poseedor de mercancías evidencian esta crisis antropológica, que bien podría ser catalogada como la falsa condena opresora a la realidad unidimensional del capitalismo global.

Es de especial interés en este trabajo focalizar la mirada en los dos primeros capítulos del texto de Freire Pedagogía Del Oprimido (1970), en cuanto es en estos dos capítulos en donde el denominado primer Freire establece los supuestos antropológico - filosóficos de su propuesta pedagógica, política y filosófica. No es de extrañar que en estas primeras páginas el autor establece 
diálogos con otras tradiciones del pensamiento crítico y humanista como el humanismo del joven Marx y la teoría crítica frankfurtiana.

Con respecto a la lectura realizada por Freire a Marx (Muñoz,2014), el pedagogo logra desarrollar en clave de una teoría crítica de la educación las tesis de Marx en su lectura crítica a la opresión en la sociedad burguesa capitalista. Freire consigue ubicar las tesis de la crítica política y económica del joven Marx en una crítica a las formas bancarias de la educación y en una propuesta educativa libertaria y progresista. Para ello se rescata la comprensión antropológica del ser humano como ser de relaciones y como especie tendiente, dada su imperfección, a tener que transformarse constantemente, dos condiciones de posibilidad antropológico - filosóficas de lo que políticamente se puede entender como revolución. Tanto para Freire como para Marx, la revolución es permanente o constante y no un resultado burocrático de la lucha social, un tema que daría para otro trabajo y que por motivos de tiempo y espacio no podrá ser desplegado en estas páginas.

Esta apropiación puede evidenciarse en citas de Freire a textos de Marx como La Sagrada Familia (1962) y Las Tesis Sobre Feuerbach (1966) en la perspectiva de poder comprender antropológica y políticamente al ser humano en su doble implicación de subjetividad (ser actuante) y objetividad (condiciones materiales de la actuación históricamente producida), unidas en una especial concepción de praxis entendida como:

Sólo en la solidaridad, en que lo subjetivo constituye con lo objetivo una unidad dialéctica, es posible la praxis auténtica. Praxis que es reflexión y acción de los hombres sobre el mundo para transformarlo. Sin ella es imposible la superación de la contradicción opresor - oprimido4.

La lectura realizada por Freire a la escuela de Frankfurt se concentra en estos dos capítulos en las obras de Erich Fromm El Corazón Del

4 FREIRE, Paulo. Pedagogía del oprimido. México: Siglo XXI, 1983, p. 43. 
Hombre (1967) y de Herbert Marcuse Eros Y Civilización (1999) y El Hombre Unidimensional (1981). El lugar común de esta apropiación es la crítica realizada por los frankfurtianos a las maneras cada vez más sutiles y colonizadoras de las modernas sociedades capitalistas a los mundos de la vida, con una creciente burocratización y monetarización de la vida cotidiana. Desde la lectura de Fromm el amor a la muerte (necrofilia) y el miedo a la libertad serían las claves recuperadas por el pedagogo para cuestionar la función alienante de la educación bancaría. En la apropiación de Marcuse se resalta el cierre instrumental de la sociedad industrial capitalista, su unidimensionalidad y con ello la perdida del potencial creador del eros; para Freire la pedagogía hegemónica es antipedagógica en tanto no es una comprensión de la educación desde el eros, sino desde la destrucción tanatológica. En esta inicial lectura a la escuela de Frankfurt el pensador brasileño actualiza la lectura de Marx en una ampliación y potenciación de su crítica contemporánea al avance global del capitalismo.

Como podrá notarse existe una sincronía política y epistémica en este campo conceptual de la teoría crítica, del joven Marx, la Escuela de Frankfurt, la obra de Sartre y la pedagogía de Freire. Podría decirse que en esta reconstrucción crítica, la opción o interés de Freire es por una postura crítica - humanista no ortodoxa, en sus palabras: radical (Runge y Muñoz,2016). Es decir, una lectura de mundo donde lo fundamental es la emancipación o liberación humana, más allá de conjuros doctrinarios. Esta perspectiva radical tendrá en la época en que escribe sus libros Educación Como Práctica De La Libertad (1969), Pedagogía Del Oprimido(1970) y El Cambio (1970) una crítica mordaz desde posturas marxistas sectarias, como las expuestas por lecturas mecanicistas y estructuralistas al marxismo, especialmente las de línea estalinista. Paradójicamente estas interpretaciones al marxismo terminan traicionando el principio fundante de la praxis crítica: la libertad del ser humano. Para estas posturas sectarias el ser humano es simplemente un ser sujetado a estructuras que lo determinan en su totalidad. Una tesis que contradice las claridades del joven Marx, las tesis de los frankfurtianos, las lecturas sartrianas y la propuesta pedagógica freireana. 
En resumen, Freire comprenderá las propuestas críticas de Marx, de los Frankfurtianos, de Sartre y la suya como radicales, es decir, centradas en la liberación humana de sus opresiones. Y las lecturas ortodoxas, estructuralistas, mecanicistas y esencialista como sectarias o centradas en la defensa dogmática de un presupuesto ideológico. En sus palabras:

La sectarización es siempre castradora por el fanatismo que la nutre. La radicalización, por el contrario, es siempre creadora, dada la criticidad que la alimenta. En tanto la sectarización es mítica, y por ende alienante, la radicalización es crítica y, por ende, liberadora. Liberadora ya que, al implicar el enraizamiento de los hombres en la opción realizada, los compromete cada vez más en el esfuerzo de transformación de la realidad concreta, objetiva. ${ }^{5}$

Desde esta perspectiva radical, el tema de la libertad es central para la comprensión antropológica, ética y política del ser humano, de allí la pertinencia del eco sartriano en la propuesta pedagógica de Freire.

\section{LA RADICAL LIBERTAD EN FREIRE: LA CONDENA A LA LIBERTAD Y LA DECISIÓN HUMANA}

La concepción radical de la libertad implica en el pensamiento de Freire, y su conexión con Sartre, la defensa de la humana condición como una condena a la libertad, es decir, por sus condiciones antropológicas la única especie que deberá asumir la responsabilidad de la libertad será la humana. En esta perspectiva el ser humano en relación con su libertad se ve envuelto en una triple implicación: en tanto ser vivo o viviente biológico, en tanto ser viviente con consciencia de ello o ser biográfico y en tanto ser viviente con consciencia y en condiciones materiales de existencia o

5 FREIRE, Paulo. Pedagogía del oprimido. México: Siglo XXI, 1983, p. 24. 
ser histórico. A continuación se argumentarán estas tres implicaciones de la humana condición y sus relaciones con la cuestión de la libertad.

En primer lugar, el ser humano como ser viviente o biológico tiene una característica común a todo lo orgánico o biótico, su inacabamiento. Mientras lo inorgánico o abiótico está limitado en su configuración, lo orgánico o vivo siempre está en expansión o movimiento, de allí que Sartre entienda al hombre como "lo que llega a ser" (Sartre, 1966,p. 139). Este primer nivel de lo orgánico es común a todas las manifestaciones de la vida y establece un principio Biológico de fuertes consecuencias políticas: la vida es expansión y movimiento. En este sentido escribe Freire:

Los oprimidos, que introyectando la "sombra" de los opresores siguen sus pautas, temen la libertad, en la medida en que ésta, implicando la expulsión de la "sombra", exigiría de ellos que "llenaran" el de la "sombra", exigiría de ellos que "Ilenaran" el "vacío" dejado por la expulsión con "contenido" diferente: el de su autonomía. El de su responsabilidad, sin la cual no serían libres. La libertad, que es una conquista y no una donación, exige una búsqueda permanente. Búsqueda que sólo existe en el acto responsable de quien lleva a cabo. Nadie tiene libertad para ser libre, sino que al no ser libre lucha por conseguir su libertad. Ésta tampoco es un punto ideal fuera de los hombres, al cual, inclusive, se alienan. No es idea que se haga mito, sino condición indispensable al movimiento de búsqueda en que se insertan los hombres como seres inconclusos ${ }^{6}$.

Para los seres humanos que radicalmente deben pertenecer a lo orgánico y no a lo inorgánico, es decir deben ser vida y no cosas, una de sus condiciones de vida es estar y ser en expansión y movimiento. En cuanto seres vivos los seres humanos somos una especie en construcción, en movimiento una suerte de Homo Viator. Esto nos relaciona con la vida sin que de ello podamos

6 FREIRE, Paulo. Pedagogía del oprimido. México: Siglo XXI, 1983, p. 37. 
desprendernos. Lo que nos particulariza en lo biótico, es que además de ser inacabados estamos llamados a asumir consciencia de ello. Veamos: lo inorgánico está acabado, todo lo orgánico es inacabado y en lo orgánico lo humano ha de tomar consciencia de su inacabamiento. Una segunda implicación de la humana condición es la consciencia del inacabamiento, de allí su libertad.

Siendo la especie humana la única manifestación orgánica que asume la consciencia de su inacabamiento, la libertad emerge como la opción siempre de base de asumirse biótico o abiótico, biótico sin consciencia o biótico con consciencia. En el primer caso el ser humano puede decidir no ser vida, limitando su existencia a simple objeto o cosa, una cosificación de la existencia. Para muchas manifestaciones de lo supuestamente humano es mejor asumir la vida como objeto, movido por fuerzas inerciales ajenas y externas a él. Pero en este caso de cosificación, es el ser humano que se da a sí mismo este atributo no siendo ella un desenlace lógico de su "naturaleza" objetual. El ser humano puede, desde su libertad, decidir ser cosa y con ello traicionar su propia posibilidad. Albergar en sí al opresor. Para Freire:

Sufren una dualidad que se instala en la "interioridad" de su ser. Descubren que, al no ser libres, no llegan a ser auténticamente. Quieren ser, más temer ser. Son ellos y al mismo tiempo son el otro yo introyectado en ellos como conciencia opresora. Su lucha se da entre ellos como conciencia opresora. Su lucha se da entre ellos mismos o ser duales. Entre expulsar o no al opresor desde "dentro" de sí. Entre desalienarse o mantenerse alienados. Entre seguir prescripciones o tener opciones. Entre ser espectadores o actores. Entre actuar o tener la ilusión de que actúan en la acción de los opresores. Entre decir la palabra o no tener voz, castrados en su poder de crear y recrear, en su poder de transformar el mundo?.

En este sentido escribe Sartre:

7 FREIRE, Paulo. Pedagogía del oprimido. México: Siglo XXI, 1983, p. 39. 
[...] la muerte viviente es estar rodeado por la eterna preocupación de los juicios, de los actos que uno no quiere cambiar. De suerte que, en verdad, como estamos vivos, quise mostrar por el absurdo la importancia que tiene en nosotros la libertad, es decir, la importancia de cambiar los actos por otros actos. No importa cual sea el círculo infernal en el cual vivimos, creo que somos libres para quebrarlo y si las gentes no lo quiebran es que también libremente permanecen en él. De tal modo que se meten libremente en el infierno ${ }^{8}$.

Este auto reconocimiento distorsionado del ser humano como cosa o muerte viviente, evidencia la presencia del opresor en sí mismo, por ello una de las primeras actividades del viviente será desalojar de sí la falsa consciencia, el engaño de la libertad que implica auto reducirse a objeto.

El ser humano también puede reconocerse como vida pero no toma consciencia de su inacabamiento y termina junto con otras manifestaciones de la vida, como las plantas y los animales, asumiendo una concepción esencialista de su existencia. Se es vida porque así es, sin mayor consciencia histórica y biográfica de ello. El ser humano como ser viviente se reduce a simple vida sin sentido, sin proyecto. Aquí la libertad emerge como decisión hacia la naturalización de la existencia, como dejarse llevar por impulsos o fuerzas instintivas sin mayor implicación ética y política. Cierto fatalismo ante la creencia en la imposibilidad de modificar lo naturalizado. Sobre el asunto dice el pedagogo:

A veces, este fatalismo, a través de un análisis superficial, da la impresión de docilidad, como algo propio de un supuesto carácter nacional, lo que es un engaño. Este fatalismo, manifestado como docilidad, es producto de una situación histórica y sociológica y no un trazo esencial de la forma de ser del pueblo?.

\footnotetext{
8 SARTRE, Jean-Paul. Un teatro de situaciones: apartado textos y entrevistas sobre las piezas. Buenos Aires: Losada, 1979, p. 183.

9 FREIRE, Paulo. Pedagogía del oprimido. México: Siglo XXI, 1983, p. 57.
} 
Este fatalismo o docilidad no se da por naturaleza biológica, es la construcción histórica de unas maneras de relacionamiento defensoras de la opresión y que deben ser comprendidas en tanto construcción humana que puede ser deconstruida o transformada. La principal estrategia cultural y política para la reproducción de dicha docilidad es la educación bancaria o digestiva, la cual consiste en dar de comer algo al oprimido, que no es más que el alimento de su propia opresión. Freire citando a Sartre dirá:

La concepción de saber de la concepción "bancaria" es, en el fondo, lo que Sartre (El hombre y las cosas) llamaría concepción "digestiva" o "alimenticia" del saber. Éste es como si fuese el "alimento" que el educador va introduciendo en los educandos, en una especie de tratamiento de engorde ${ }^{10}$.

En segundo lugar, el ser humano como viviente que tiene consciencia de su inacabamientos se hace biográfico, no sólo posee vida sino que le asigna un sentido un proyecto. Aquí toma fuerza la concepción de proyecto de Sartre en Freire, en cuanto el ser humano no es sólo un devenir, sino que es además un devenir con sentido. Esta idea de proyecto potencia la condición humana más allá de su simple adaptación a lo existente, trasciende su mera inmersión. En las palabras de Ernani María Fiori:

La esencia humana cobra existencia autodescubriéndose como historia. Pero esa conciencia histórica, al objetivarse, se sorprende reflexivamente a sí misma, pasa a decirse, a tornarse conciencia historiada; y el hombre es conducido a escribir su historia. Alfabetizarse es aprender a leer esa palabra escrita en que la cultura se dice, y diciéndose críticamente, deja de ser repetición intemporal de lo que pasó, para temporalizarse, para concienciar su temporalidad constituyente,

10 FREIRE, Paulo. Pedagogía del oprimido. México: Siglo XXI, 1983, p. 79. 
que es anuncio y promesa de lo que ha de venir. El destino, críticamente, se recupera como proyecto"1.

El ser humano como ser proyectado hace de su viaje una constante transformación del mundo, sus relaciones y sí mismo. Aquí está la conexión fundamental entre la perspectiva crítica de Sartre y de Freire, se entiende la libertad humana como la decisión del ser humano de hacerse responsable de los efectos de su inacabamiento. El ser humano es una especie en expansión, que a diferencia de la expansión de otras especies, ha de hacerse responsable de su trayecto y proyecto. En este sentido escribe Freire:

La educación Problematizadora no es una fijación reaccionaria, es futuro revolucionario. De ahí que sea profética y, como tal, esperanzada. De ahí que corresponda a la condición de los hombres como seres históricos y a su historicidad. De ahí que se identifique con ellos como seres más allá de sí mismos; como "proyectos"; como seres que caminan hacia adelante, que miran al frente; como seres a quienes la inmovilidad amenaza de muerte; para quienes el mirar hacia atrás no debe ser una forma nostálgica de querer volver sino una mejor manera de conocer lo que está siendo, para construir mejor el futuro. De ahí que se identifique con el movimiento permanente en que se encuentran inscritos los hombres, como seres que se saben inconclusos; movimiento que es histórico y que tiene su punto de partida, su sujeto y su objeto ${ }^{12}$.

Por último, el ser humano como ser histórico comprende su ser viviente y biográfico siempre en condiciones materiales de existencia, que no son determinaciones sino condiciones de posibilidad. Tanto para Sartre como para Freire, claramente influenciados por Marx, las condiciones

\footnotetext{
1 FREIRE, Paulo. Pedagogía del oprimido. México: Siglo XXI, 1983, p.16.

12 FREIRE, Paulo. Pedagogía del oprimido. México: Siglo XXI, 1983, p. 93.
} 
materiales u objetivas son la presentación actual de la larga actividad humana, el presente es el cumulo de trabajo desplegado por la especie humana y que se actualiza en cuanto puede ser afectada por la actividad de los contemporáneos. Las condiciones materiales se entienden como condiciones de posibilidad dado que actualizan las energías utópicas humanas, sus prácticas de libertad que permiten introducir a las condiciones preexistentes nuevas actuaciones y contenidos. Lo instituido por la acción pasada es instituyente en la acción presente. En palabras del pedagogo:

Si lo que caracteriza a los oprimidos, como "conciencia servil", en relación con la conciencia del señor, es hacerse "objeto", es transformarse, como señala Hegel, en "conciencia para otro", la verdadera solidaridad con ellos está en luchar con ellos para la transformación de la realidad objetiva que los hace "ser para otro"3.

Esta comprensión histórica del ser viviente y biográfico reta política y éticamente al ser humano a hacer algo con lo que los otros hicieron de él. Una idea de praxis que combina la reflexividad, la actividad y la transformación de las situaciones de opresión que imposibilitan el llamado histórico del ser humano de ser más.

\section{CONCLUSIONES}

Se ha procurado en este texto exponer, grosso modo, las principales implicaciones de la apropiación realizada por Freire a Sartre en su concepción radical de libertad. Por ello, la idea de libertad en ambos autores se sumerge en la complejidad de la humana condición para desprender desde lo biológico, biográfico e histórico una defensa de la libertad como condena o supuesto de partida de toda arquitectura antropológica.

13 FREIRE, Paulo. Pedagogía del oprimido. México: Siglo XXI, 1983, p. 40. 
A diferencia de otras orientaciones teóricas y políticas, que como el estructuralismo, defienden como base antropológica la sujetación o la sujeción, esta lectura crítica se fundamenta en la defensa de la humana condición como posibilidad de perfectibilidad humana, lo que Freire entenderá como "vocación ontológica de ser más" (1983, p. 32) y Sartre como "condena a la libertad". Sartre en su obra El Existencialismo Es Un Humanismo afirmaba que:

\begin{abstract}
El hombre es ahora absolutamente libre. Pero esta libertad no es un don, sino que el hombre se halla condenado a ella, condenado porque para que la libertad sea plena libertad, no puede haber nada enfrentado normativamente al hombre, ni fe en Dios, ni verdades, ni valores ${ }^{14}$.
\end{abstract}

La actual pertinencia de esta apropiación y por ello de la obra del filosofo y del pedagogo radica en su potencial para develar las situaciones de opresión actuales y sus juegos ideológicos de "falsa libertad". A diferencia de fascismos anteriores, como los de Estado, los actuales fascismos no se muestran como tales, sus nuevas mascaras son contradictoriamente las de la libertad. Después de las experiencias dictatoriales del siglo XX ningún nuevo fascismo se muestra como tal. Los nuevos fascismos de mercado ya no se erigen en el control evidente y sus implicaciones biopolíticas, ahora sus estrategias y tácticas se despliegan enunciando libertades y emancipaciones que confunden la crítica ¿Qué se podría criticar de un tipo de orden social que ya ha conquistado la libertad y la emancipación humana?, ese parece ser su conjuro ideológico. De allí que volver a las obras de estos autores permita vivificar el potencial de la crítica como desnaturalización, como develamiento de los juegos ideológicos aturdidores. Y claro está, en consecuencia con ello asumir el llamado a una praxis revolucionaria que sea capaz, en su constancia y movimiento, de des - institucionalizar la opresión dominante, desmontando sus disfraces libertarios.

14 SARTRE, Jean-Paul. El existencialismo es un humanismo. Buenos Aires: Losada, 1998, p. 11-12. 


\section{REFERENCIAS BIBLIOGRÁFICAS}

CHARTIER, Roger et al. Cultura escrita, literatura e historia: coacciones transgredidas y libertades restringidas. Conversaciones de Roger Chartier. 2. ed. México: Fondo de Cultura Económica, 2000.

ESTRADA LOPERA, Luis Esteban. Fenomenología y humanismo en la pedagogía de Paulo Freire. Kénosis, Rionegro, v. 6, n. 11, p. 17-45, 2018.

FREIRE, Paulo. La educación como práctica de la libertad. México: Siglo XXI, 1971. FREIRE, Paulo. Pedagogía del oprimido. México: Siglo XXI, 1983.

FROMM, Erich. El corazón del hombre. México: Fondo de Cultura Económica, 1967. MARCUSE, Herbert. El hombre unidimensional. Barcelona: Planeta Agostini, 1981. MARCUSE, Herbert. Eros y civilización. Barcelona: Ariel, 1999.

MARX, Karl. Tesis sobre Feuerbach. Moscú: Editorial Progreso, 1966.

MARX, Karl. Contribución a la crítica de la filosofía del derecho de Hegel. In: MARX, Karl. Escritos de juventud sobre el derecho (Textos 1837-1847). Barcelona: Anthropos, 2008.

MARX, Karl; ENGELS, Frederic. La sagrada familia y otros escritos. México: Grijalbo, 1962.

MUÑOZ GAVIRIA, Diego Alejandro. Antropología filosófica relacional en la obra de Paulo Freire: diálogos con la teoría crítica de Karl Marx. Revista Perseitas, [s. l.], v. 2, n. 2, p. 186-203, 2014.

RUNGE-PEÑA, Andrés Klaus; MUÑOZ, Diego Alejandro. La antropología pedagógica 'radical' de Paulo Freire: formabilidad, inacabamiento, formación y educación del ser humano. In: OSPINA, Héctor Fabio; RAMÍREZ-LÓPEZ, Camilo Andrés (comp.). Pedagogía crítica latinoamericana y género. Bogotá: Siglo Del Hombre, 2016.

SARTRE, Jean-Paul. El hombre y las cosas. Buenos Aires: Losada, 1965.

SARTRE, Jean-Paul. El ser y la nada. Buenos Aires: Losada, 1966.

SARTRE, Jean-Paul. La nausea. Buenos Aires: Losada, 1975.

SARTRE, Jean-Paul. Un teatro de situaciones: apartado textos y entrevistas sobre las piezas. Buenos Aires: Losada, 1979.

SARTRE, Jean-Paul. El existencialismo es un humanismo. Buenos Aires: Losada, 1998. 
INTUITIO | PORTO ALEGRE | V. 12, N. 2, JUL.-DEZ. 2019 | e-

\section{Endereço Correspondência:}

Sector 3, cra. 46 No. 40B 50, Rionegro, Antioquia, Colômbia 